\title{
Evaluación del Error Dimensional en la Reproducción Tridimensional de una Mandíbula Humana Disecada Mediante Prototipado Rápido de Modelado por Deposición Fundida
}

\author{
Evaluation of the Dimensional Error in the Three-Dimensional Reproduction of \\ a Dissected Human Jaw by Rapid Prototyping of Molten Deposition Modeling
}

Constanza Salas Salas; Luis Araneda Silva \& Sebastian Schott Börger

\begin{abstract}
SALAS, S. C.; ARANEDA, S. L. \& SCHOTT, B. S. Evaluación del error dimensional en la reproducción tridimensional de una mandíbula humana disecada mediante prototipado rápido de modelado por deposición fundida. Int. J. Odontostomat., 14(1):5-11, 2020.

RESUMEN: Las técnicas de prototipado rápido se están utilizando de forma masiva en distintas áreas médicas, siendo de gran utilidad para el manejo preoperatorio y para el ahorro de tiempo clínico en diversos procedimientos quirúrgicos. El propósito de este trabajo fue fabricar un biomodelo mediante impresión 3D utilizando la técnica de modelado por deposición fundida, corroborando que fuese una réplica fiel de una mandíbula humana disecada. Se generó un modelo 3D a partir de la adquisición volumétrica con CBCT de una mandíbula con marcadores craneométricos de referencia y se imprimió utilizando técnica de modelado por deposición fundida. La comparación de ambas mandíbulas se realizó con la medición de las distancias entre los distintos puntos craneométricos, de forma homóloga. No hubo diferencia estadística para los resultados de la comparación entre ambas estructuras, presentando un error dimensional promedio de $0,16 \pm 0,11$. El modelo obtenido corresponde a una réplica fidedigna de la mandíbula original, permitiendo validar la técnica para posteriores fines clínicos.
\end{abstract}

PALABRAS CLAVE: mandíbula, cirugía oral, tomografía computada cone-beam, modelo anatómico, impresión tridimensional.

\section{INTRODUCCIÓN}

El prototipado rápido $(\mathrm{RP})$, corresponde a la técnica que incluye distintas tecnologías de obtención de estructuras desde un "diseño asistido por computador" (CAD), en pocas horas y con poca intervención humana (Pham \& Gault, 1998). El resultado obtenido es una representación sólida tridimensional del diseño desarrollado digitalmente. El perfeccionamiento del prototipado rápido está estrechamente relacionado con el avance computacional, siendo esencial la presencia de un sistema CAD para su fabricación (Ghane, 2016).

El RP puede ser aplicado en múltiples circunstancias, por ejemplo, en su uso doméstico o industrial. Pero adquiere importante valor su relación con el área médica al desarrollar biomodelos a partir de datos obtenidos del escáner de estructuras anatómicas mediante tomografía computada, resonancia magnética o láser (Petzold et al., 1999).

Dentro de las aplicaciones de esta tecnología de RP en el área de la salud, se encuentran (Le et al., 2005):

Diseño y manufactura de biomodelos, herramientas de utilización quirúrgica e implantes.

Modelos de entrenamiento quirúrgico y dispositivos médicos que cumplen roles importantes en el perfeccionamiento técnico de profesionales de áreas médicas tanto en pregrado como postgrado. 
SALAS, S. C.; ARANEDA, S. L. \& SCHOTT, B. S. Evaluación del error dimensional en la reproducción tridimensional de una mandíbula humana disecada mediante prototipado rápido de modelado por deposición fundida. Int. J. Odontostomat., 14(1):5-11, 2020.

Plataformas en aplicaciones de ingeniería de tejidos. Estas superficies son utilizadas para el cultivo y crecimiento de tejido celular ex vivo.

De esta forma el prototipado rápido nos permite adquirir biomodelos que pueden ser de tipo (Lohfeld et al., 2005):

- Virtual: corresponde a la imagen en 3D obtenida a partir de la adquisición volumétrica de una estructura esqueletal mediante, por ejemplo, un escáner de tomografía computada.

- Computacional: creado con el propósito de desarrollar un análisis del comportamiento biomecánico de una estructura biológica.

- Físico: es una estructura sólida producida por tecnología de ingeniería y que usualmente, su diseño proviene de un biomodelo computacional.

Debemos obtener una imagen virtual para fabricar una estructura sólida, por lo que es necesario captar la figura mediante un sistema radiológico, en donde se ha visto mayor preponderancia de la tomografía de haz cónico (cone beam) en los últimos años, en el ámbito odontológico. A pesar de las ventajas que presentaría la tomografía computada en el área médica, el cone beam tiene una serie de ventajas que han permitido masificar su uso, entre ellos la menor exposición a radicación, alta resolución y mejor rendimiento en zona de dientes y hueso alveolar (De Vos et al., 2009; European Commission, 2012; Varga et al., 2013).

Una vez obtenido el archivo DICOM de la adquisición volumétrica, es necesario traspasar la información a un formato STL a partir de la técnica de conversión de datos llamada segmentación de imagen en donde se obtiene la geometría estructural superficial de la imagen como un entramado similar a un mosaico (Kragskov et al., 1996).

El procesamiento de la imagen puede realizarse de forma manual o automática a partir de un software especializado y así obtener la estructura virtual deseada, pero a menudo se requiere una combinación entre segmentación automatizada y manual debido a la complejidad de las regiones anatómicas y de la presencia de artefactos que modifican el umbral de intensidad (Huotilainen et al., 2014).

Existen distintos tipos de obtención mecánica de un RP, dependiendo del tipo de material que se utilice, que se pueden clasificar en técnicas de adición de capas, de sustracción y de deformación de material. Dentro de las técnicas de adición de capas encontramos la técnica de modelado por deposición fundida (FDM) en donde un polímero fundido, habitualmente utilizado acrinonitrilo butadieno diestireno o poliácido láctico, es depositado en capas sobre una superficie en la que se suelda en frío. El modelado por deposición fundida es muy adecuado para la fabricación de réplicas de hueso, ya que utiliza materiales que elaboran prototipos duros y robustos, así como también reproduce estructuras de forma compleja como guías y modelos quirúrgicos (Negi et al., 2014).

Distintos tipos de impresoras pueden ser encontradas en el mercado, catalogándose además de su método de prototipado rápido, por su costo. Hoy en día se ha producido una gran expansión de las máquinas de bajo costo, relacionado con la necesidad de transferir estas nuevas tecnologías a un dominio público, pero es necesario validar su exactitud dimensional, para que puedan ser utilizadas sin peligro en el área médica (Maschio et al., 2016). Una de las impresoras tridimensionales categorizadas como low-cost es la impresora Cube Pro® que permite construir modelos de hasta 285,4 × 270,4 x 230 $\mathrm{mm}$ de dimensión con un solo cabezal. Con relación a la resolución, se puede obtener un espesor mínimo de capa de un grosor de 70 micrones en su opción de mayor calidad.

Con respecto a la exactitud dimensional de los biomodelos, ésta se debe evaluar antes de decidir la aplicabilidad de la estructura, pudiendo producirse errores dimensionales en las distintas etapas de la fabricación del prototipo (Choi et al., 2002), por lo que en la literatura se describe una exactitud dimensional aceptable, por lo menos clínicamente, cuando el porcentaje de error en modelado por adición de capas se encuentra entre el 0,6 y el $6 \%$. Esto puede aplicarse para la mayoría de los usos del prototipado rápido, pero es de crucial importancia para modelos craneomaxilofaciales con los que se requiere una aún más alta precisión cuando estos son utilizados para la fabricación de guías de implantes (Huotilainen et al.).

El objetivo de este trabajo de investigación es calcular la variación dimensional del biomodelado de prototipado rápido por deposición fundida en la reproducción de una mandíbula humana disecada, con el fin de comprobar que presentan una exactitud dimensional equivalente. 


\section{MATERIAL Y MÉTODO}

Para efectuar la investigación se realizó un proceso de adquisición volumétrica o registro tridimensional en la Facultad de Odontología de la Universidad de Chile, donde se utilizó para dichos efectos el equipo de CBCT Planmeca Promax 3D Mid Proface con el protocolo 3D Dental-Cara. Este formato tiene una zona colimada (FOV: Field of view) de $160 \mathrm{~mm}$ de diámetro y $160 \mathrm{~mm}$ de altura. Los parámetros de exposición para dicho sistema fueron de $90 \mathrm{kv}, 12 \mathrm{mAs}$ durante 27 segundos.

La toma del volumen se realizó utilizando los parámetros de exposición indicados por el fabricante para una mandíbula humana natural, sobre una mandíbula humana disecada de adulto, a la que se le instalaron marcadores radiopacos, ubicados en puntos craneométricos definidos. Dicha mandíbula fue facilitada por el departamento de Anatomía y Morfología de la Facultad de Medicina de la Universidad de Chile y
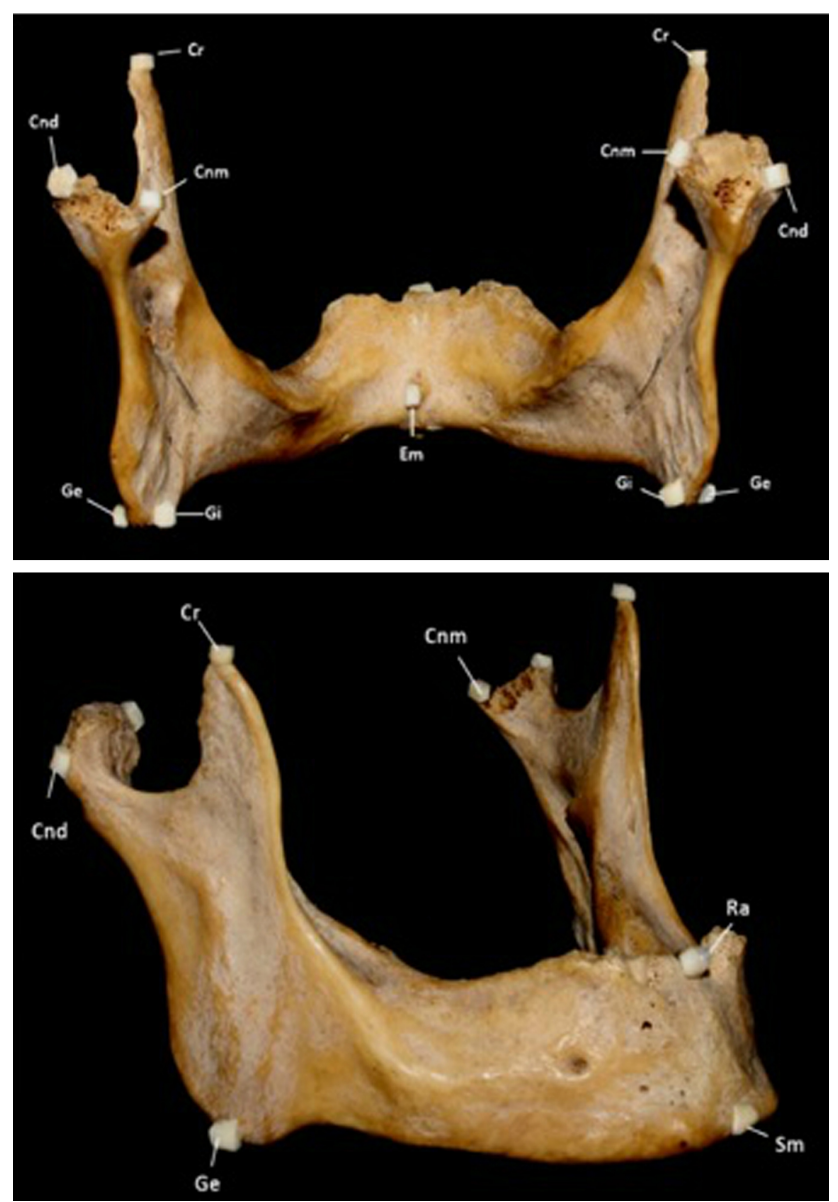

Fig. 1. Distribución de los marcadores en sus respectivos puntos craneométricos. los marcadores fueron obtenidos mediante el corte de tejido dental, de dientes donados por pacientes sometidos a cirugía de terceros molares con el debido consentimiento informado, hasta obtener sólo la dentina y se ubicaron sobre puntos de referencia anatómicos clásicos (Fig. 1). La mandíbula se dispuso sobre el equipo en un pedestal que lo acopló con el mismo.

Una vez adquirido el volumen, éste se manipuló con el software Romexis Viewer® para su validación crítica, lo que fue realizado por un Radiólogo Dento Máxilofacial del Servicio de Radiología de la Facultad de Odontología de la Universidad de Chile. Esto implicó definir los límites de visualización del examen, en el cual el total del FOV incluyó completamente la zona de interés.

Habiendo realizado la validación critica, el volumen se exportó a un dispositivo de almacenamiento portable en formato DICOM, mediante el cual fue llevado a la Facultad de Arquitectura y Urbanismo de la Universidad de Chile. En aquel lugar se manipuló digitalmente para obtener un biomodelo virtual. Una vez que se obtuvo la imagen tridimensional el siguiente paso correspondió a eliminar las superficies que no tenían contacto con el volumen mayor en el registro del Cone Beam CT manteniendo la mayor cantidad de detalles y luego se configuraron los pilares para el soporte de la impresión. Realizada la eliminación de superficies, el volumen se exportó en formato STL para ser codificado por la impresora 3D Cube Pro Duo $\AA$, en ella se seleccionaron parámetros de calidad de terminación media, con un grosor de capa de 0,07 mm. Luego de la obtención del modelo, se retiraron los pilares de soporte y se pulieron las espículas remanentes hasta obtener superficies suaves al tacto (Fig. 2).

Una vez fabricado el biomodelo físico, la inspección visual del prototipo obtenido reveló superficies del cóndilo que no fueron reproducidas, situación que se discutirá más adelante, razón por la que se modificó el punto Cdm a una posición ubicada a $56 \mathrm{~mm}$ de $\mathrm{Gi}$, de forma bilateral tanto para la mandíbula como para el modelo. Posteriormente se sometió a la comparación de las distancias entre los marcadores radiopacos de la mandíbula disecada, según el estudio realizado por Ibrahim et al. (2009), con sus correspondientes mediciones homólogas en la estructura impresa. Este trabajo experimental se llevó a cabo en el Servicio de Radiología 

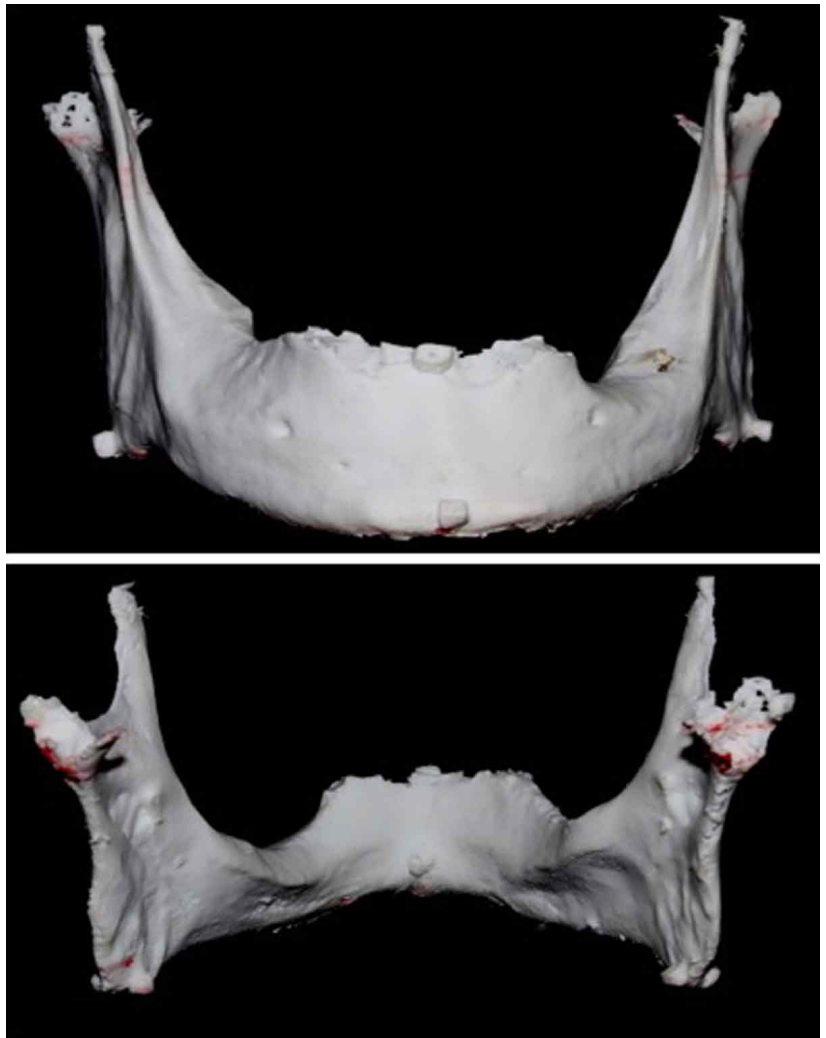

Fig. 2. Vistas frontal y dorsal, respectivamente, del resultado de la impresión tridimensional.

Dentomaxilar de la Facultad de Odontología de la Universidad de Chile, durante un periodo de 20 días.

Una vez realizadas las mediciones para cada prototipo (mandíbula seca y modelo) se procedió al análisis estadístico de sus resultados; de manera específica se centró en el análisis descriptivo e inferencial. La manera de proceder fue la siguiente:
La descripción del error dimensional se realizó con dos análisis homólogos, uno para la mandíbula aislada y otro para su respectivo biomodelo físico. Se procedió a efectuar 21 mediciones de las distancias comprendidas entre los distintos marcadores como se indica en la Tabla I, tomadas desde el centro de cada uno de ellos, tanto para la mandíbula como para el prototipo, con la excepción particular de las distancias: $\mathrm{Cnm}-\mathrm{Cr}$ de forma bilateral y $\mathrm{Cnm}-\mathrm{Cnm}$, debido a la modificación de $\mathrm{Cnm}$ descrita anteriormente. En total se obtuvieron 42 mediciones registradas cada una de ellas con un calibrador digital marca Kamasa ${ }^{\circledR}$, modelo KM-448 con 3 estrellas de calidad.

El modelo de experimentación involucró que cada una de las 21 mediciones se repitió 30 veces por el mismo observador, tanto para la mandíbula como para su modelo, esto con el fin de analizar y medir la incertidumbre.

\section{RESULTADOS}

Para cada una de las distancias registradas (21), tanto en la mandíbula como en el modelo, se procedió a estudiar la distribución de los errores; para esto se obtuvo diferentes medidas de resumen, en particular: el promedio, su desviación estándar y su índice de varianza. Posteriormente se realizó un test de muestras independientes desde el punto de vista paramétrico (test para cociente de varianza y test para diferencia de medias) para analizar si hubo diferencia estadística significativa de las medias entre los dos grupos, esto es entre la mandíbula seca y el prototipo, considerando un nivel de significancia del $5 \%$.

Tabla I. Identificación de las distancias de referencia que se utilizaron para la comparación de los biomodelos.

\begin{tabular}{lc}
\hline Distancias bilaterales (a ambos lados de la mandíbula) & $\mathbf{C n d}-\mathbf{G e}$ \\
\hline Polo distal del cóndilo - Ángulo mandibular externo (Altura de la rama mandibular) & $\mathbf{C n m}-\mathbf{G i}$ \\
Polo medial del cóndilo - Ángulo mandibular interno & $\mathbf{G e ~ - ~ C r}$ \\
Ángulo mandibular externo - Proceso coronoides & $\mathbf{G i}-\mathbf{C r}$ \\
Ángulo mandibular interno - Proceso coronoides & $\mathbf{C n d ~ - ~ C r}$ \\
Polo distal del cóndilo - Proceso coronoides & $\mathbf{C n m}-\mathbf{C r}$ \\
Polo medial del cóndilo - Proceso coronoides & $\mathbf{G e ~ - ~ S m ~}$ \\
Ángulo mandibular externo - Sínfisis mentoniana (Largo del cuerpo mandibular) & $\mathbf{G i ~ - ~ E m ~}$ \\
Ángulo mandibular interno - Espinas mentonianas & $\mathbf{C r}-\mathbf{R a}$ \\
Proceso coronoides - Reborde alveolar medial & \\
Distancias únicas & $\mathbf{C n d ~ - ~ C n d ~}$ \\
Distancia intercondílea externa & $\mathbf{C n m}-\mathbf{C n m}$ \\
Distancia intercondílea interna & $\mathbf{G i ~ - ~ G i ~}$ \\
Distancia intergoníaca &
\end{tabular}


SALAS, S. C.; ARANEDA, S. L. \& SCHOTT, B. S. Evaluación del error dimensional en la reproducción tridimensional de una mandibula humana disecada mediante prototipado rápido de modelado por deposición fundida. Int. J. Odontostomat., 14(1):5-11, 2020.

En el test de cociente de varianza se utilizó la prueba de Levene que es una prueba estadística inferencial que se usa para evaluar la igualdad de la varianza mediante un intervalo de confianza entre las mediciones de una variable calculada, en este caso, para dos grupos. Por otra parte, el test de diferencia de medias o test- $T$, genera el intervalo de confianza a partir de los promedios de las muestras.

De los resultados del análisis estadístico se puede desprender que según los intervalos de confianza obtenidos se podría ratificar parcialmente la hipótesis, ya que sólo en los resultados para Cnm-Gi de forma bilateral de la prueba de diferencia de medias el valor$P$ es menor a 0,05 , existiendo diferencia estadística sólo en estas dos mediciones específicas, debido posiblemente a que no se encontró de forma constante y clara la longitud interna de ambas ramas mandibulares (distancia Gi-Cnm) en el modelo, efecto esperado que se discutirá más adelante. Estos resultados no interfieren en el resultado general del análisis en base a los promedios de los datos obtenidos, siendo $p>$ a 0,05.

Para cada medición lineal se determinó la desviación media absoluta como la diferencia absoluta entre cada medida registrada en el modelo con su respectivo resultado en la mandíbula seca. A partir de esto se obtuvo la diferencia media relativa con el fin de establecer la discrepancia porcentual de los resultados. Los datos obtenidos nos entregaron una diferencia dimensional promedio de 0,160 $\pm 0,114 \mathrm{~mm}$ y porcentualmente, valores que varían entre el $0,002 \%$ y el $0,72 \%$.

\section{DISCUSIÓN}

En nuestra comparación del biomodelo creado a partir de una mandíbula disecada, encontramos una buena reproducción global de las dimensiones registradas entre los distintos puntos craneométricos. Según los resultados obtenidos, existe un rango promedio de variación longitudinal entre la mandíbula disecada y el modelo de 0,16 $\pm 0,114 \mathrm{~mm}$, con un índice de significancia media medido por el valor $p$ de 0,674 y 0,561 a partir de las pruebas estadísticas realizadas. El valor máximo en relación con la desviación media absoluta obtenida fue de 0,38 $\pm 0,273 \mathrm{~mm}$ y el mínimo fue de 0,002 $\pm 0,001 \mathrm{~mm}$.

Para explicar la falta de precisión en la reproducción de los cóndilos mandibulares, la evidencia dice que la elección del umbral de segmentación puede causar la pérdida de información en áreas donde sólo hay presencia de hueso muy delgado (Winder \& Bibb, 2005). Esto coincide con nuestros resultados, aquellas zonas que no estaban cubiertas por hueso cortical no aparecen en el modelo o fueron cubiertas al momento del procesamiento de la imagen, debido a que el entramado cubre completamente la superficie externa. Esto explica que distancias como la altura interna de la rama $(\mathrm{Cnm}-\mathrm{Gi})$ no hayan podido ser bien definidas en la reproducción.

Con respecto a la comparación entre los resultados de otros estudios, la mayoría de la evidencia científica muestra fabricación de modelos mediante estereolitografía (SLA), pero son empleados de igual forma para estos efectos ya que los usos de los biomodelos son similares, independiente de su proceso de fabricación.

Kouhi et al. (2008) realizaron comparaciones dimensionales de una mandíbula humana disecada y sus prototipos obtenidos mediante técnica de FDM a partir de una adquisición en CT helicoidal. Si bien sus resultados no pueden ser utilizados para nuestra comparación ya que midieron contornos y profundidades estructurales, tomaron en cuenta el grado de resolución de capas necesario para obtener un modelo fidedigno, concluyendo que con un grosor de capas de $0,4 \mathrm{~mm}$ se puede obtener la precisión suficiente para la aplicación clínica en cirugía mandibular y maxilofacial (Kouhi et al.). En nuestro estudio el grosor de capas obtenido fue de $0,07 \mathrm{~mm}$, por lo que se sitúa dentro del rango de precisión esperado.

Finalmente, durante el año 2015, Federico Maschio probó la exactitud dimensional de una impresora 3D que utiliza, al igual que en nuestro estudio, la técnica de FDM y para la adquisición volumétrica también utilizó CBCT. Para el estudio replicaron dos mandíbulas humanas disecadas dentadas y compararon cada una con su respectivo modelo en distancias determinadas. Dentro de sus resultados obtuvieron distintas discrepancias al considerar aquellas longitudes menores o mayores a $12 \mathrm{~mm}$. Su desviación media relativa para aquellas distancias más pequeñas fue de un 3,76 \%, en cambio para las más grandes fue de $1,09 \%$. Cabe señalar que en nuestro estudio todas las distancias registradas eran mayores a $12 \mathrm{~mm}$, por lo que sería interesante investigar en el futuro el comportamiento dimensional del modelo en base a una mandíbula dentada en la que se pueda obtener puntos constantes de comparación, como lo realizó el autor (Maschio et al.). 
Nuestras evaluaciones indican que sería factible utilizar estos prototipos para fines quirúrgicos, por lo que sería recomendable realizar pruebas en base CBCT de individuos vivos con el fin de analizar el comportamiento de aquellas zonas de hueso delgado, como los cóndilos.

\section{CONCLUSIONES}

Concluimos que la variación dimensional general de la técnica de FDM es de 0,16 mm lo que representa un $0,21 \%$, por lo que se considera posible obtener un biomodelo anatómico con exactitud dimensional suficiente para ser utilizado con fines clínicos, a partir del uso de una impresora de bajo costo como la 3D Cube Pro Duo $囚$. Las discrepancias en las mediciones efectuadas entre mandíbula seca y biomodelo oscilan entre $0,002 \mathrm{~mm}$ y $0,56 \mathrm{~mm}$ lo que representa variaciones entre el $0,002 \%$ y el $0,72 \%$ que se encuentran dentro de lo esperado en base a los resultados encontrados en la literatura.

La diferencia estadística entre las dimensiones obtenidas fue de 0,674 y 0,571 para los análisis de cociente de varianza y T-test realizados, respectivamente. Lo que indica que no existe diferencia estadística entre los valores registrados en la mandíbula disecada y su modelo.

Finalmente, un hallazgo importante de esta investigación es la utilización de marcadores radiopacos fabricados con dentina, que permitió disminuir la presencia de artefactos en la adquisición volumétrica y realizar mediciones confiables en segmentos definidos tanto en la mandíbula original como en su reproducción tridimensional.

A partir de los resultados del presente estudio se sugiere realizar pruebas en estructuras de conformación ósea más compleja, como huesos esfenoides y temporal para considerar su utilización con fines académicos, así como también la evaluación de la fabricación de estructuras de alta definición como las guías de implantes quirúrgicos, para su posterior aplicación clínica.

SALAS, S. C.; ARANEDA, S. L. \& SCHOTT, B. S. Evaluation of the dimensional error in the three-dimensional reproduction of a dissected human jaw by rapid prototyping of molten deposition modeling. Int. J. Odontostomat., 14(1):5-11, 2020.
ABSTRACT: Rapid prototyping techniques are widely used in different medical areas, being useful for preoperative management and saving clinical time in various surgical procedures. The purpose of this work was the manufacture of a biomodel by the means of 3D printing using the fused deposition modeling technique, proving that it was a faithful replica of a dissected human jaw. A 3D model was generated from the volumetric acquisition with CBCT of a mandible with craniometric reference markers and was printed using fused deposition modeling technique. The comparison of both jaws was made with the measurement of the distances between the different craniometric points, in a homologous way. There was no statistical difference for the results of the comparison between both structures, obtaining an average dimensional error of $0.16 \pm 0.11$. The manufactured model corresponds to a reliable replica of the original jaw, allowing validation of the technique for later clinical purposes.

KEY WORDS: mandible, oral surgery, cone-beam computed tomography, anatomic models.

\section{REFERENCIAS BIBLIOGRÁFICAS}

Choi, J. Y.; Choi, J. H.; Kim, N. K.; Kim, Y.; Lee, J. K.; Kim, M. K.; Lee, J. H. \& Kim, M. J. Analysis of errors in medical rapid prototyping models. Int. J. Oral Maxillofac. Surg., 31(1):23-32, 2002.

De Vos, W.; Casselman, J. \& Swennen, G. R. Cone-beam computerized tomography (CBCT) imaging of the oral and maxillofacial region: a systematic review of the literature. Int. J. Oral Maxillofac. Surg., 38(6):609-25, 2009.

European Commission. Cone Beam CT for Dental and Maxillofacial Radiology. Evidence-Based Guidelines. Radiation Protection $N^{\circ}$ 172. Luxembourg, Directorate-General for Energy, Directorate D - Nuclear Energy, Unit D4 - Radiation Protection, 2012.

Ghane, D. B.. Rapid prototyping: the revolutionary technology and applications review. Int. J. Innov. Eng. Res. Technol., 3(2), 2016. Available from: https://www.ijiert.org/admin/papers/ 1457532069_Volume \%203\%20Issue \%202.pdf

Huotilainen, E.; Jaanimets, R.; Valásek, J.; Marcián, P.; Salmi, M.; Tuomi, J.; Mäkitie, A. \& Wolff, J. Inaccuracies in additive manufactured medical skull models caused by the DICOM to STL conversion process. J. Craniomaxillofac. Surg., 42(5):e25965, 2014

Ibrahim, D.; Broilo, T. L.; Heitz, C.; de Oliveira, M. G.; de Oliveira, H. W.; Nobre, S. M.; Dos Santos Filho, J. H. \& Silva, D. N. Dimensional error of selective laser sintering, three-dimensional printing and PolyJet models in the reproduction of mandibular anatomy. J. Craniomaxillofac. Surg., 37(3):167-73, 2009.

Kouhi, E.; Masood, S. \& Morsi, Y. Design and fabrication of reconstructive mandibular models using fused deposition modeling. Assem. Autom., 28(3):246-54, 2008.

Kragskov, J.; Sindet-Pedersen, S.; Gyldensted, C. \& Jensen, K. L. A comparison of three-dimensional computed tomography scans and stereolithographic models for evaluation of craniofacial anomalies. J. Oral Maxillofac. Surg., 54(4):402-11, 1996.

Le, C. H.; Zlatov, N.; Sloten, J. V.; Bohez, E.; Khanh, L.; Binh, P. H.; Oris, P. \& Toshev, Y. 2005. Medical rapid prototyping applications and methods. Assem. Autom., 25(4):284-92, 2005. 
SALAS, S. C.; ARANEDA, S. L. \& SCHOTT, B. S. Evaluación del error dimensional en la reproducción tridimensional de una mandíbula humana disecada mediante prototipado rápido de modelado por deposición fundida. Int. J. Odontostomat., 14(1):5-11, 2020.

Lohfeld, S.; Barron, V. \& McHugh, P. E. Biomodels of bone: a review. Ann. Biomed. Eng., 33(10):1295-311, 2005.

Maschio, F.; Pandya, M. \& Olszewski, R. Experimental validation of plastic mandible models produced by a "low-cost" 3-dimensional fused deposition modeling printer. Med. Sci. Monit., 22:943-57, 2016

Negi, S.; Dhiman, S. \& Sharma, R. K. Basics and applications of rapid prototyping medical models. Rapid Prototyp. J., 20(3):256-67, 2014.

Petzold, R.; Zeilhofer, H. F. \& Kalender, W. A. Rapid protyping technology in medicine--basics and applications. Comput. Med. Imaging Graph., 23(5):277-84, 1999.

Pham, D. T. \& Gault, R. S. A comparison of rapid prototyping technologies. Int. J. Mach. Tools Manuf., 38(10-11):1257-87, 1998.

Varga, E. Jr.; Hammer, B.; Hardy, B. M. \& Kamer, L. The accuracy of three-dimensional model generation. What makes it accurate to be used for surgical planning? Int. J. Oral Maxillofac. Surg., 42(9):1159-66, 2013.

Winder, J. \& Bibb, R. Medical rapid prototyping technologies: state of the art and current limitations for application in oral and maxillofacial surgery. J. Oral Maxillofac. Surg., 63(7):100615, 2005.
Dirección para correspondencia:

Sebastian Schott Börger

Escuela de Graduados

Facultad de Odontología

Universidad de Chile

Av. Santa María 0596

Providencia

Santiago

CHILE

Email: sschott@odontologia.uchile.cl

Recibido : 23-01-2019

Acpetado: 05-09-2019 